\title{
PREDICTING PRODUCTIVITY LOSS CAUSED BY CHANGE ORDERS USING THE EVOLUTIONARY FUZZY SUPPORT VECTOR MACHINE INFERENCE MODEL
}

\author{
Min-Yuan CHENG $^{\text {a }}$, Dedy Kurniawan WIBOWO ${ }^{\mathrm{a}, \mathrm{b}}$, Doddy PRAYOGO ${ }^{\mathrm{a}}$, \\ Andreas F. V. ROY \\ ${ }^{a}$ Department of Civil and Construction Engineering, National Taiwan University of Science and Technology, \\ \#43, Sec. 4, Keelung Rd., Taipei, Taiwan, R. O.C. \\ ${ }^{b}$ Department of Civil Engineering, Sepuluh Nopember Institute of Technology, Nopember, Indonesia \\ ${ }^{c}$ Department of Civil Engineering, Faculty of Engineering, Parahyangan Catholic University, \\ Jalan Ciumbuleuit 94, Bandung, 40141 West Java, Indonesia
}

Received 07 Dec 2012; accepted 19 Feb 2013

\begin{abstract}
Change orders in construction projects are very common and result in negative impacts on various project facets. The impact of change orders on labor productivity is particularly difficult to quantify. Traditional approaches are inadequate to calculate the complex input-output relationship necessary to measure the effect of change orders. This study develops the Evolutionary Fuzzy Support Vector Machines Inference Model (EFSIM) to more accurately predict change-order-related productivity losses. The EFSIM is an AI-based tool that combines fuzzy logic (FL), support vector machine (SVM), and fast messy genetic algorithm (fmGA). The SVM is utilized as a supervised learning technique to solve classification and regression problems; the FL is used to quantify vagueness and uncertainty; and the fmGA is applied to optimize model parameters. A case study is presented to demonstrate and validate EFSIM performance. Simulation results and our validation against previous studies demonstrate that the EFSIM predicts the impact of change orders significantly better than other AI-based tools including the artificial neural network (ANN), support vector machine (SVM), and evolutionary support vector machine inference model (ESIM).
\end{abstract}

Keywords: change orders, productivity loss, fuzzy logic, support vector machine, fast messy genetic algorithm.

\section{Introduction}

Changes during construction projects are very common, making construction one of the most complex industries. Changes can involve adding to or reducing the scope of project work or correcting or modifying an original design. Change orders in the construction industry have negative effects in aspects such as cost, quality, time, and organization. While most change order items (e.g. material, scheduling, rework, equipment) can be relatively easy to measure, quantifying the impact on labor productivity is typically more complicated (Hanna et al. 1999a).

Many studies have reported on the impact of change orders on labor productivity. The methods used in the literature to calculate productivity loss can be grouped into the 3 categories of (1) regression analysis (Leonard 1988; Moselhi et al. 1991; Ibbs 2005), (2) artificial neural network (ANN) (Moselhi et al. 2005), and (3) statisticalfuzzy (Hanna et al. 2002). Previous studies (Hanna et al. 2002; Moselhi et al. 2005) have reported that ANN and statistical-fuzzy methods outperform regression analysis. However, no method is suitable for calculating productivity loss because prediction accuracies are outside of acceptable limits.

Construction projects are complex undertakings full of uncertainty and vagueness. Developing a deterministic mathematical model to predict productivity loss is difficult and expensive. An inference model (Cheng, Wu 2009) offering high accuracy and low cost is one feasible approach to predicting productivity loss. Inference models derive new facts from historical data. The human brain can learn previous information and deduce new facts from that information. Artificial intelligence (AI) can be employed to develop models that simulate human brain functions. AI is concerned with computer systems able to handle complex problems using techniques such as Artificial Neural Network (ANN), Support Vector Machine (SVM), and Fuzzy Logic (FL). AI-based inference models thus offer a promising solution to predicting productivity loss.

Several AI hybrid systems have been developed in recent years that have solved various construction management problems (Cheng, Wu 2009; Cheng, Roy 2010).

Corresponding author: Doddy Prayogo

E-mail: doddyprayogo@ymail.com 
In an AI hybrid system, fusing different AI techniques can achieve better results than a single AI technique because the advantages of one technique can compensate for another's disadvantages (Cheng, Wu 2009). The Evolutionary Fuzzy Support Vector Machine Inference Model (EFSIM) (Cheng, Roy 2010) was proposed to further improve prediction accuracy. EFSIM is an artificial intelligence (AI) hybrid system that fuses fuzzy logic (FL), support vector machine (SVM), and fast messy genetic algorithm (fmGA). In EFSIM, FL deals with vagueness and uncertainty; SVM acts as a supervised learning tool; and fmGA works to optimize FL and SVM parameters. EFSIM significantly reduces the level of human intervention and can be used by professionals who do not have background in AI (Cheng, Roy 2010).

The objective of this research is to use EFSIM to predict productivity loss caused by change orders. Feasibility and capability of the proposed method are evaluated and compared with other methods, including ANN, SVM, and ESIM (Cheng, Wu 2009). Validation with previous studies (Moselhi et al. 2005) is also carried out to demonstrate proposed model performance.

\section{Literature review}

\subsection{Productivity loss caused by change orders}

Change can be defined as any modification in the original scope, time, or cost of the work (Hester et al. 1991). A change order is issued to formally announce the change and modify the contract between the contractor and owner (Hester et al. 1991). Keane et al. (2010) grouped causes of change into four categories: owner-related, consultant-related, contractor-related, and non-partyrelated, and effects of change into five categories: cost-related, quality-related, time-related, organizationrelated, other effects (Keane et al. 2010).

Preliminary research into calculating the effects of change orders on labor productivity was accomplished by Leonard (1988). This research attempted to identify the effects of change orders on labor productivity in 90 cases facing change-order-related productivity losses. Results indicated a significant correlation between change orders and productivity loss. However, there were limitations to Leonard's study, including limited number of variables and subjective evaluation (Hanna et al. 1999a, b). This preliminary study motivated other researchers to develop research in this field further.

Two studies used a statistical method to quantify the impact of change orders on labor productivity in mechanical and electrical construction projects (Hanna et al. 1999a, b). These studies used the delta method as an efficiency indicator and regression analysis to analyze questionnaire data. Hanna et al. (2002) improved the method by using the statistical-fuzzy technique to quantify the cumulative impact of change orders. Unfortunately, the technique is difficult for stakeholders to implement due to complicated calculation steps and poor prediction results.
A neural network model (Moselhi et al. 2005) was developed to estimate the impact of change orders on labor productivity, including the timing effect of change orders. Analysis results showed this model estimated the impact of change orders on productivity more accurately than those previously described. However, this method could gain better prediction results by fusing the neural network model with an AI technique.

\subsection{Fuzzy logic (FL)}

FL is a popular AI technique invented by Zadeh in the 1960s. FL has been used in forecasting, decision making, and action control in environments characterized by uncertainty, vagueness, presumptions, or subjectivity (Bojadziev, G., Bojadziev, M. 2007). In general, FL systems have four major components: fuzzification, fuzzy rule base, inference engine, and defuzzification. Fuzzification is a process that uses membership functions (MFs) to convert the value of each input variable into a corresponding linguistic variable degree. Fuzzy rules represent relations between input and output fuzzy sets and form the basis for fuzzy logic to obtain fuzzy output. The result of fuzzification, which is used by the inference engine, stimulates the human decision-making process based on fuzzy implications and available rules. Lastly, defuzzification reverses the fuzzification process and converts the fuzzy set into crisp output.

The advantages of FL related to vagueness and uncertainty depend heavily on the appropriate distribution of membership functions (MFs), number of rules, and selection of proper fuzzy set operations. Greater problem complexity increases the difficulty of MF construction and rules (Ko 2002). Some researchers have treated this drawback as an optimization problem because determining MF configurations and fuzzy rules is complicated and problem-oriented. To overcome such difficulties, some researchers have tried to fuse FL with AI optimization techniques such as GA and ant colony (Ishigami et al. 1995; Martinez et al. 2008). These optimization methods have demonstrated their ability to minimize timeconsuming operations and reduce the level of human intervention necessary to optimize MFs and fuzzy rules.

\subsection{Support vector machine (SVM)}

SVM (Vapnik 1995) is an AI paradigm already used in a wide range of applications. SVM is a learning tool for solving classification and regression problems. SVM works by plotting input vectors into a higher dimensional feature space. The optimal hyperplane is identified within this feature space with the help of a kernel function, $\mathrm{K}\left(\mathrm{x}_{\mathrm{i}}, \mathrm{x}_{\mathrm{j}}\right)$. A radial basis function (RBF) kernel has been recommended for general users as a first choice due to its ability to analyze higher-dimension data, use of only one hyperparameter in searches, and fewer numerical difficulties (Hsu et al. 2003).

SVM has achieved performance levels comparable to or higher than traditional learning tools (Burges 
1998; Yongqiao et al. 2005). However, SVM's generalization ability and prediction accuracy are determined by the optimal penalty $(C)$ and kernel $(\gamma)$ parameters. To overcome this drawback, an optimization technique (e.g. fmGA) may be used to identify the optimum values of parameters simultaneously (Cheng, Wu 2009).

\subsection{Fast messy genetic algorithm (fmGA)}

fmGA is a recently developed machine learning and optimization tool based on a genetic algorithm approach (Goldberg et al. 1993). fmGA is an improvement on messy genetic algorithms (mGAs), which were initially developed to overcome linkage problems in simple genetic algorithms (sGAs) resulting from a parameter coding problem that sometimes generates suboptimal solutions (Deb, Goldberg 1991). Unlike sGAs, which use fixed length strings to represent possible solutions, fmGA applies messy chromosomes to form strings of various lengths that can efficiently find optimal solutions for large-scale permutation problems (Feng, Wu 2006).

The fmGA contains two loop types: inner and outer (Fig. 1). The process starts with the outer loop. Firstly, a competitive template (randomly generated or problemspecific) is generated. In the inner loop, the fmGA operation is three-phase, including an initialization phase, primordial phase, and juxtapositional phase. In the initialization phase, an adequately large population contains all possible building blocks (BBs) of order $k$. fmGA performs the probabilistic complete initialization (PCI) by generating $n$ chromosomes randomly and evaluating their fitness value. The primordial phase contains two operations, namely threshold selection and building-block filtering. In this phase, "bad" genes that do not belong to BBs are filtered out so that, in the end, the result encloses a high proportion of "good" genes belonging to BBs. In the juxtapositional phase, fmGA operations are similar to sGA operations. The selection for "good" genes is used together with a cut-and-splice operator to form a high-quality generation that may contain the optimal solution. The next outer loop begins after the respective inner loop is finished. The competitive template is replaced by the best solution found so far, which becomes the new competitive template for the next outer loop. The whole process is performed until the

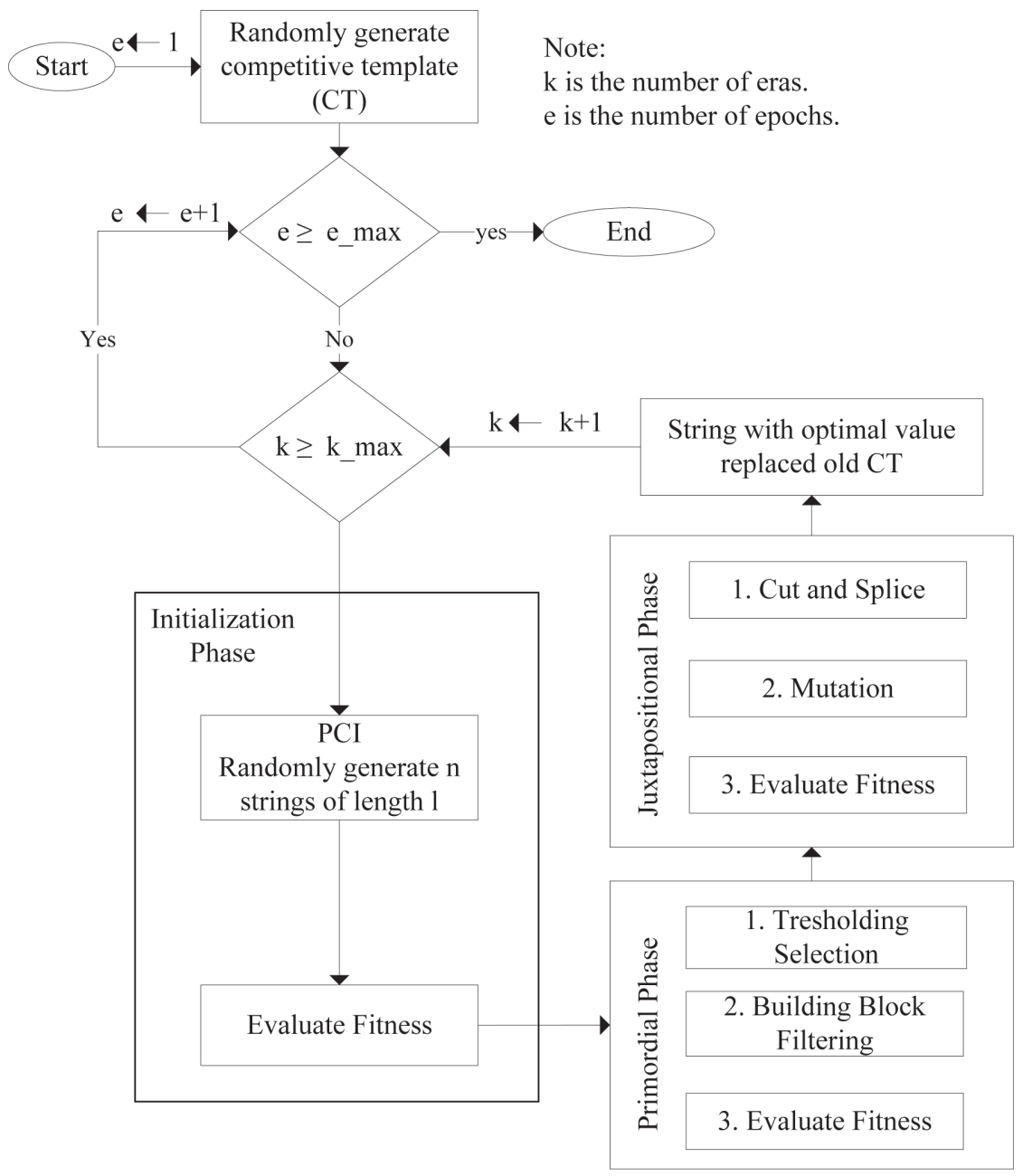

Fig. 1. Illustration of fmGA 
maximum number of eras $\left(k_{\max }\right)$ is reached. The fmGA can also be performed over epochs $\left(e_{\max }\right)$. An epoch is the complete process between first era and the maximum number of eras $\left(k_{\max }\right)$. Epochs can be performed as many times as desired. The algorithm is terminated once a good-enough solution is obtained or no further improvement is made.

\section{Evolutionary fuzzy support vector machine inference model}

The evolutionary fuzzy support vector machine inference model (EFSIM) is a hybrid AI system developed by Cheng and Roy (2010) that fuses the three different AI techniques of fuzzy logic (FL), support vector machine (SVM), and fast messy genetic algorithm (fmGA). In this complementary system, FL deals with vagueness and approximate reasoning; SVM acts as a supervised learning tool to handle fuzzy input-output mapping; and fmGA works to optimize FL and SVM parameters.

In EFSIM, the fuzzy inference engine and fuzzy rules based on the FL system have been replaced by SVM. However, SVM's generalization ability and prediction accuracy are determined by the optimal penalty $(C)$ and kernel $(\gamma)$ parameters. Improper tuning of the parameters will affect the accuracy of the prediction model. To overcome this shortcoming, EFSIM utilizes fmGA to search simultaneously for optimum SVM parameters and FL parameters. The architecture of EFSIM is shown in Figure 2.

The EFSIM involves eight major steps, beginning with training data and ending with the optimal prediction model. An explanation of major steps involved in EFSIM is given below:

1) Training data

Final data for training are obtained from data preprocessing output. Data preprocessing used in this study included data cleaning, attribute reduction and data transformation.

\section{2) Fuzzification}

Each normalized input attribute from the previous step is converted into membership grades corresponding to the specific membership function (MF) set generated and encoded by fmGA. This model uses trapezoidal and triangular MF shapes (see Fig. 3) that, in general, may be developed by referencing summit points and widths. This study used the Summit and Width Representation Method (SWRM) (Ko 2002) to encode complete MF sets (Fig. 3 (c)). Figure 4 illustrates the fuzzification process.

3) SVM training model

SVM addresses the complex relationship between fuzzy input and output variables. Fuzzification process output, in the form of membership grades, is fuzzy input for SVM. SVM trains the dataset to obtain the prediction model, with penalty $(C)$ and kernel $(\gamma)$ as its parameters, which are randomly generated and encoded by fmGA. This study used the RBF kernel as a reasonable first choice (Hsu et al. 2003).

4) Defuzzification

This is a fuzzification reverse process. Once SVM finishes the training process, output numbers are expressed in terms of a fuzzy set. Output numbers are then converted into crisp numbers. Employing fmGA, the model generates a random defuzzification parameter $(d f p)$ substring and encodes it for conversion into SVM fuzzy output. This evolutionary approach is simple and straightforward, as it uses $d f p$ as a common denominator for SVM output.

5) fmGA parameter search

fmGA is utilized to search simultaneously the fittest shapes for MFs, $d f p$, penalty parameter $(C)$, and RBF kernel parameter $(\gamma)$. In fmGA, the chromosome that represents the possible solution for searched parameters consists of four parts: the MFs substring, $d f p$ substring, penalty parameter substring, and kernel parameter substring (Cheng, Roy 2010). The chromosome is encoded into a binary string. Chromosomes consist of two segments: FL and SVM. Figure 5 illustrates the chromosome

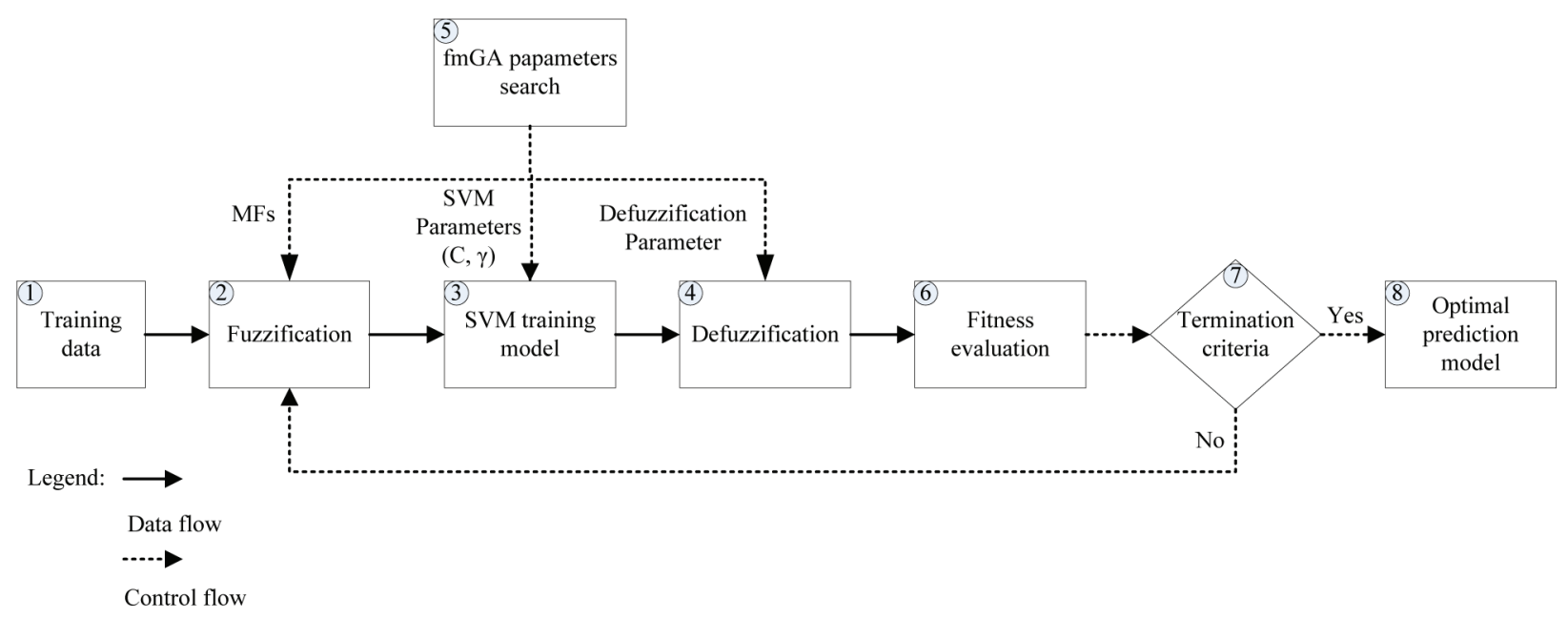

Fig. 2. Architecture of EFSIM 


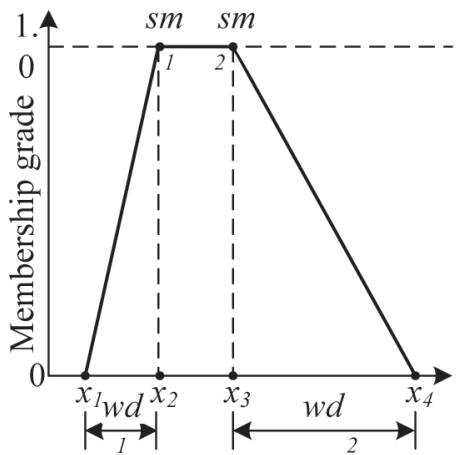

(a)

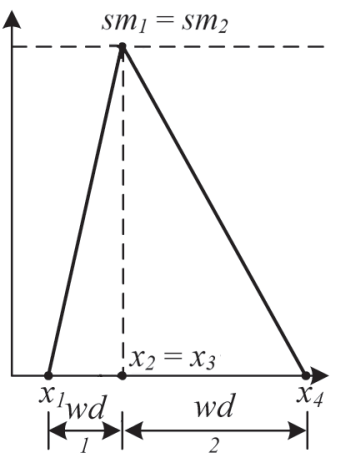

(b)

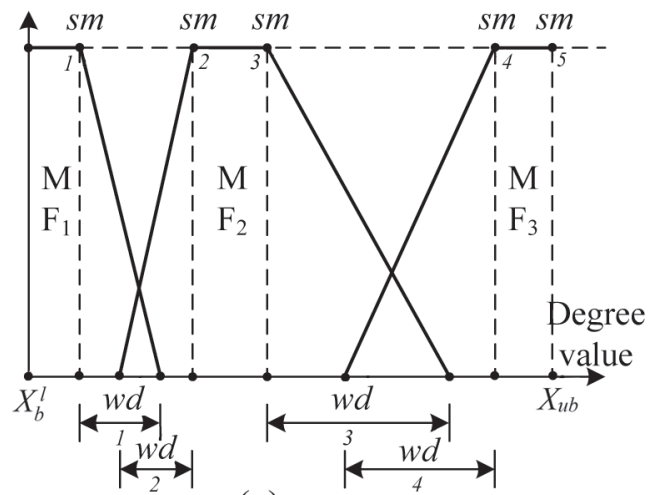

(c)

Fig. 3. Membership function: trapezoidal (a), triangular (b), complete MF set (c) (Ko 2002)

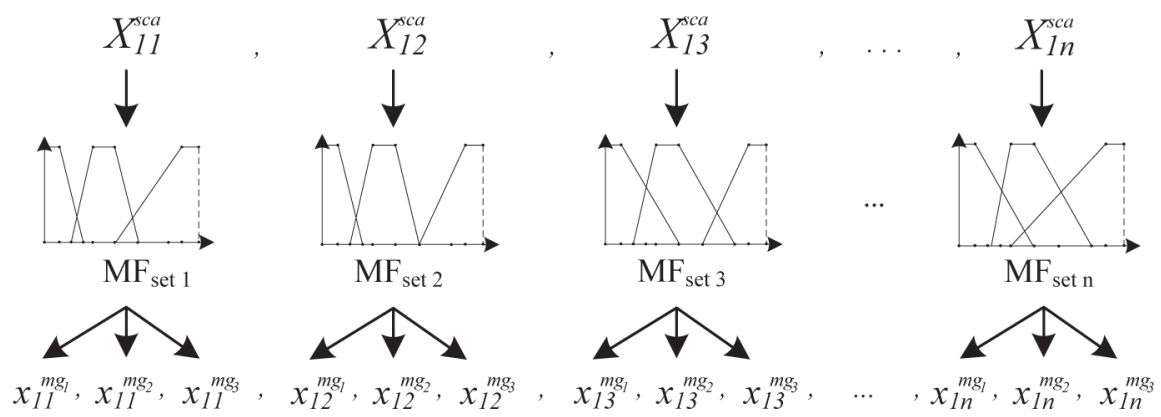

Legend:

$\begin{array}{ll}X_{i j}^{s c a}: \text { scaled input pattern } & i \text { : number of cases } \\ x_{i j}^{m g_{k}}: \text { membership grade } k \text { of } X_{i j}^{s c a} & j: \text { number of input pattern }\end{array}$

$k$ : number of MFs in one complete membership set

Fig. 4. Fuzzification process

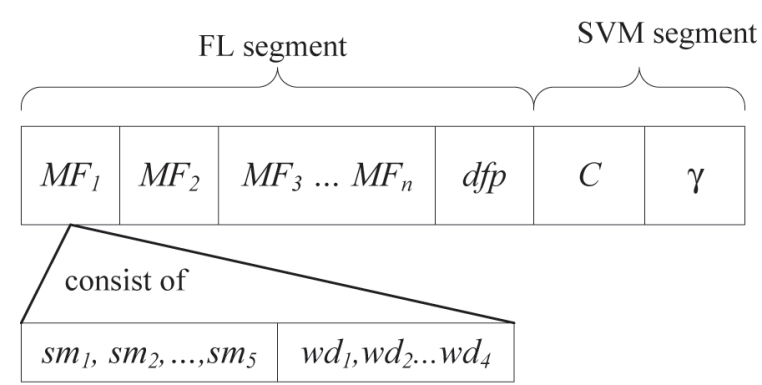

Legend:

$M F_{i}$ : membership function $i$-th

$d f p:$ defuzzification parameter

$C$ : penalty parameter

$\gamma:$ RBF kernel parameter

$s m_{j}$ : summit point $j$-th of $M F_{i}$

$w d_{j}$ : width $j$-th of $M F_{i}$

Fig. 5. EFSIM chromosome structure

structure. Table 1 summarizes the parameter settings and number of bits required for the chromosome design. For more details about fmGA parameter search, readers are referred to the previous work of Cheng and $\mathrm{Wu}$ (2009) and Cheng and Roy (2010).
Table 1. Summary of EFSIM parameter settings

\begin{tabular}{lccc}
\hline Parameter & $\begin{array}{r}\text { Upper } \\
\text { bound }\end{array}$ & Lower bound & $\begin{array}{c}\text { Number of } \\
\text { bits }\end{array}$ \\
\hline MF set & - & - & $27^{\mathrm{a}}$ \\
\hline$C$ & 200 & 0 & 5 \\
\hline$\gamma$ & 1 & 0.0001 & 10 \\
\hline$d f p$ & 1 & 0.5 & 9 \\
\hline
\end{tabular}

a Number of bits required for one complete MF set.

6) Fitness evaluation

A fitness function, a function designed to measure model accuracy and good generalization properties (Ko 2002), is now developed to evaluate fitness value. This function describes the fittest shape of MFs, optimized $d f p$ number, and SVM parameters. The fitness function consists of parameters to calculate accuracy and model complexity, as expressed in Eqn (1):

$$
f^{f i}=\frac{1}{c^{a w} \times s^{e r}+c^{c w} \times m c},
$$

where $c^{a w}$ represents the accuracy weighting coefficient; $s^{e r}$ represents the prediction error between actual output 
and desired output; $c^{c w}$ represents the complexity weighting coefficient; and $m c$ represents model complexity, which can be quantified by counting the number of support vectors.

7) Termination criteria

The process terminates when the termination criterion is satisfied. While still unsatisfied, the model will proceed to the next generation. As EFSIM uses fmGA, the termination criterion used in this study was either era number $(k)$ or epoch number $(e)$.

8) Optimal prediction model

The loop stops when the termination criterion is fulfilled. This condition means that the prediction model has identified the input/output mapping relationship with optimal C, $\gamma$, and $d f p$ parameters and is ready to predict new facts.

\section{Productivity loss prediction using EFSIM}

\subsection{Historical data}

Data used in this research were drawn from 102 cases cited in Assem's thesis (2000) and covered 33 cases from Assem's (2000) investigation of the adverse effects of change orders and 69 cases from Leonard's (1988) investigation of change order impacts. A summary of cases is shown in Table 2.

\subsection{Data preprocessing}

Data preprocessing is an important stage in data analysis that resolves the "unclean" nature of real-world data (Zhang et al. 2003). Several data preprocessing techniques such as data cleaning, attributes reduction, and data transformation were employed in this study. A systematic

Table 2. Summary cases

\begin{tabular}{|c|c|c|c|}
\hline Parameter & Min & Max & Mean \\
\hline Work type & \multicolumn{3}{|c|}{$\begin{array}{l}\text { (Architectural, Civil, Electrical, } \\
\text { Mechanical) }\end{array}$} \\
\hline Change orders number & 7 & 2150 & 106.784 \\
\hline $\begin{array}{l}\text { Frequency of change } \\
\text { orders a }\end{array}$ & 0.389 & 195.454 & 8.614 \\
\hline $\begin{array}{l}\text { Average size of change } \\
\text { orders } b\end{array}$ & 3.214 & 1595.238 & 161.964 \\
\hline Change orders hours & 100 & 83000 & 10528.688 \\
\hline $\begin{array}{l}\text { Change orders hours } \\
\text { ratio to the planned } \\
\text { hours }\end{array}$ & 0.009 & 2.660 & 0.323 \\
\hline $\begin{array}{l}\text { Change orders hours } \\
\text { ratio to the actual hours }\end{array}$ & 0.006 & 0.573 & 0.198 \\
\hline Type of impact & \multicolumn{3}{|l|}{$(1,2, \text { or } 3)^{\mathrm{c}}$} \\
\hline \multicolumn{4}{|c|}{$\begin{array}{l}\text { a frequency of change order: ratio change orders number to } \\
\text { the actual duration in months; } \\
\text { b average size of change orders: ratio of change orders hours } \\
\text { to the number of change orders; } \\
\text { c } 1 \text { represents change-order causes of productivity loss only; } \\
2 \text { or } 3 \text { represents change order plus } 1 \text { or } 2 \text { additional major } \\
\text { causes of productivity loss. }\end{array}$} \\
\hline
\end{tabular}

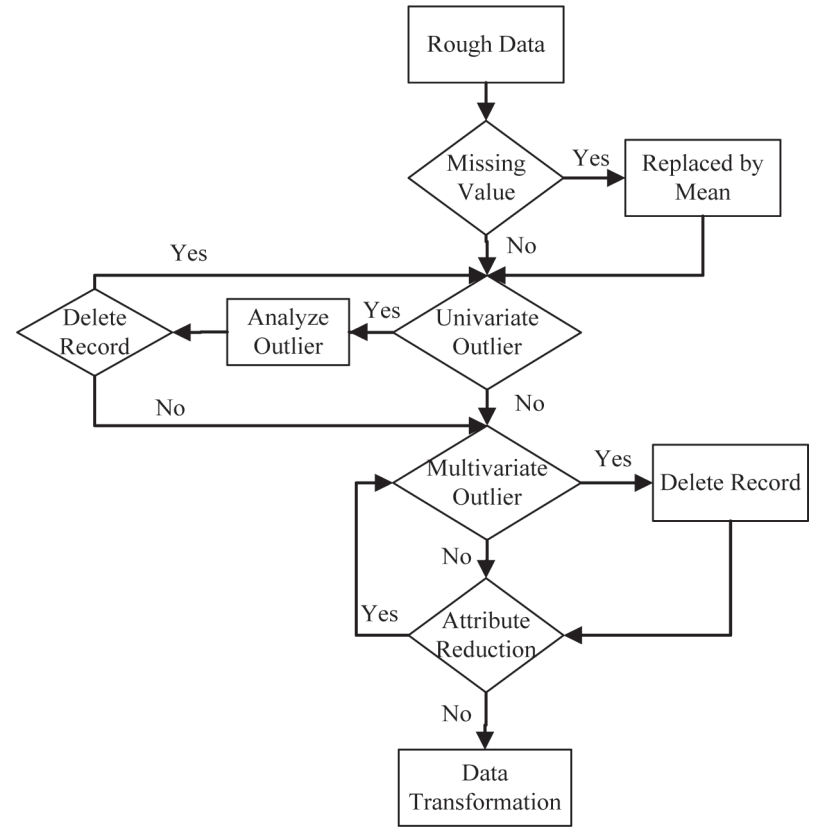

Fig. 6. Flowchart of data preprocessing

data-preprocessing flowchart (Fig. 6) was developed to obtain better prediction results. Historical data was analyzed using this flowchart to obtain training data.

Data cleaning can be applied to fill in missing values and remove noisy data (univariate and multivariate outliers) (Han, Kamber 2007; Shahi et al. 2009). Attributes reduction was applied to reduce the dimensionality of data attributes and help reduce computational time by eliminating unnecessary attributes. Two methods, correlation analysis (CA) and principal component analysis (PCA), were employed to compare attributes reduction method results. CA is the simplest way to assess input-output relationships. PCA is used to identify strong predictor variables in a dataset. Data transformation techniques such as normalization, where attribute data are scaled to fall within a small specified range, may improve the accuracy and the efficiency of mining algorithms involving distance measurements (Han, Kamber 2007; Shahi et al. 2009). The function used to normalize data in this study is shown in Eqn (2):

$$
x_{\text {norm }}=\frac{\left(x_{i}-x_{\min }\right)}{\left(x_{\max }-x_{\min }\right)},
$$

where: $x_{\text {norm }}$ is the normalized data; $x_{i}$ is the observed data; $x_{\min }$ is the minimum data; and $x_{\max }$ is the maximum data.

\subsection{Final data}

A total of 96 records were used to train the prediction model. Two kinds of analyses were done to compare performance of attribute reduction methods. Analysis 1 used CA to reduce attributes and Analysis 2 used PCA to do the same. As shown in Table 3, CA and PCA identified 6 and 4 attributes, respectively, as significant factors. Both analyses transformed the data into values ranging 
Table 3. Summary of training data

\begin{tabular}{|c|c|c|}
\hline & Analysis 1 (CA) & Analysis 2 (PCA) \\
\hline \multirow{6}{*}{ 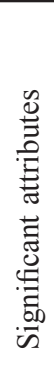 } & Work type & Work type \\
\hline & Frequency of change orders & Type of impact \\
\hline & $\begin{array}{l}\text { Average size of change } \\
\text { orders }\end{array}$ & $\begin{array}{l}\text { Frequency of change } \\
\text { orders }\end{array}$ \\
\hline & Change order hours & Time related \\
\hline & $\begin{array}{l}\text { Change order hours ratio to } \\
\text { the planned hours }\end{array}$ & $\begin{array}{l}\text { Relative size of change } \\
\text { orders }\end{array}$ \\
\hline & $\begin{array}{l}\text { Change order hours ratio to } \\
\text { the actual hours }\end{array}$ & \\
\hline
\end{tabular}

between 0 and 1 . Table 4 shows example input and output data from Analysis 1.

\subsection{Cross-validation}

Cross-validation is a statistical technique that assesses how accurately a predictive model will perform by dividing data into two segments, of which one is used to learn or train the model and the other is used to test or validate the model. 10-fold cross-validation resulted in the best performance in the simulation (Borra, Di Ciaccio 2010). In 10-fold cross-validation, original data was randomly portioned into 10 equally (or approximately equally) sized segments. Consequently, 10 independent performance estimations of training and testing were performed such that, within estimation, a different fold of the data was alternately used for testing while the remaining 9 folds were used for training (Fig. 7). We then calculated the average of each performance measure to obtain crossvalidation accuracy.

\subsection{Performance measures}

This research used the following four performance measures to evaluate EFSIM:

1. Root mean square error

Root mean square error (RMSE) is the square root of the average squared distance of predicted values by the model and the observed values. RMSE can be used to calculate the variation of errors in a prediction model and is very useful when large errors are undesirable. The RMSE is expressed using the following equation:

$$
R M S E=\sqrt{\frac{1}{n} \sum_{j=1}^{n}\left(y_{j}-\hat{y}_{j}\right)^{2}},
$$

where $y_{j}$ is the actual value; $\hat{y}_{j}$ is the predicted value; and $n$ is the number of data samples.

2. Mean absolute error

Mean absolute error (MAE) is the average absolute value of the residual (error). MAE is a quantity used to measure how close forecasts or predictions are to eventual outcomes. The MAE is expressed using the following equation:

$$
M A E=\frac{1}{n} \sum_{j=1}^{n}\left|y_{j}-\hat{y}_{j}\right| .
$$

\begin{tabular}{|c|c|c|c|c|c|c|c|}
\hline No. & $\begin{array}{c}\text { Actual \% } \\
\text { productivity } \\
\text { loss }\end{array}$ & Work type & Frequency & Average size & $\begin{array}{c}\text { Change order } \\
\text { hours }\end{array}$ & $\begin{array}{c}\text { Change order } \\
\text { hours/planned } \\
\text { hours }\end{array}$ & $\begin{array}{c}\text { Change order } \\
\text { hours/actual } \\
\text { hours }\end{array}$ \\
\hline 1 & 23.7 & 1 & 1.54545 & 33.41176 & 568 & 0.08686 & 0.06494 \\
\hline 2 & 24.5 & 1 & 1.36000 & 165.02941 & 5611 & 0.11567 & 0.08492 \\
\hline 3 & 31.8 & 1 & 1.20000 & 69.75000 & 837 & 0.13368 & 0.08745 \\
\hline 4 & 11 & 3 & 9.75000 & 25.35897 & 989 & 0.08074 & 0.07122 \\
\hline 5 & 27 & 3 & 0.47619 & 138.80000 & 1388 & 0.68850 & 0.42382 \\
\hline 6 & 19 & 3 & 1.81818 & 71.15625 & 5692.5 & 0.12291 & 0.09728 \\
\hline$\ldots$ & $\ldots$ & $\ldots$ & $\ldots$ & $\ldots$ & $\ldots$ & $\ldots$ & $\ldots$ \\
\hline 41 & 18.4 & 2 & 12.04762 & 129.64427 & 32800 & 0.41362 & 0.33745 \\
\hline 42 & 22.81 & 2 & 21.11111 & 113.15789 & 21500 & 0.48864 & 0.37719 \\
\hline 43 & 14.18 & 2 & 0.80000 & 191.66667 & 2300 & 0.10000 & 0.08582 \\
\hline 44 & 17.75 & 2 & 0.73333 & 272.72727 & 3000 & 0.05769 & 0.04745 \\
\hline 45 & 19.03 & 2 & 0.71429 & 190.00000 & 1900 & 0.13333 & 0.10795 \\
\hline 46 & 21.05 & 2 & 6.25000 & 189.33333 & 14200 & 0.13524 & 0.10677 \\
\hline$\ldots$ & $\ldots$ & $\ldots$ & $\ldots$ & $\ldots$ & $\ldots$ & $\ldots$ & $\ldots$ \\
\hline 92 & 38.71 & 4 & 34.00000 & 135.29412 & 23000 & 0.68047 & 0.39792 \\
\hline 93 & 44.31 & 4 & 4.64286 & 61.53846 & 4000 & 0.66116 & 0.53121 \\
\hline 94 & 48.95 & 4 & 6.47059 & 156.36364 & 17200 & 1.01176 & 0.51652 \\
\hline 95 & 35.09 & 4 & 2.42857 & 63.23529 & 4300 & 0.11406 & 0.07713 \\
\hline 96 & 45.34 & 4 & 2.92857 & 336.58537 & 13800 & 0.31364 & 0.17143 \\
\hline
\end{tabular}

Table 4. Input and output data for Analysis 1 


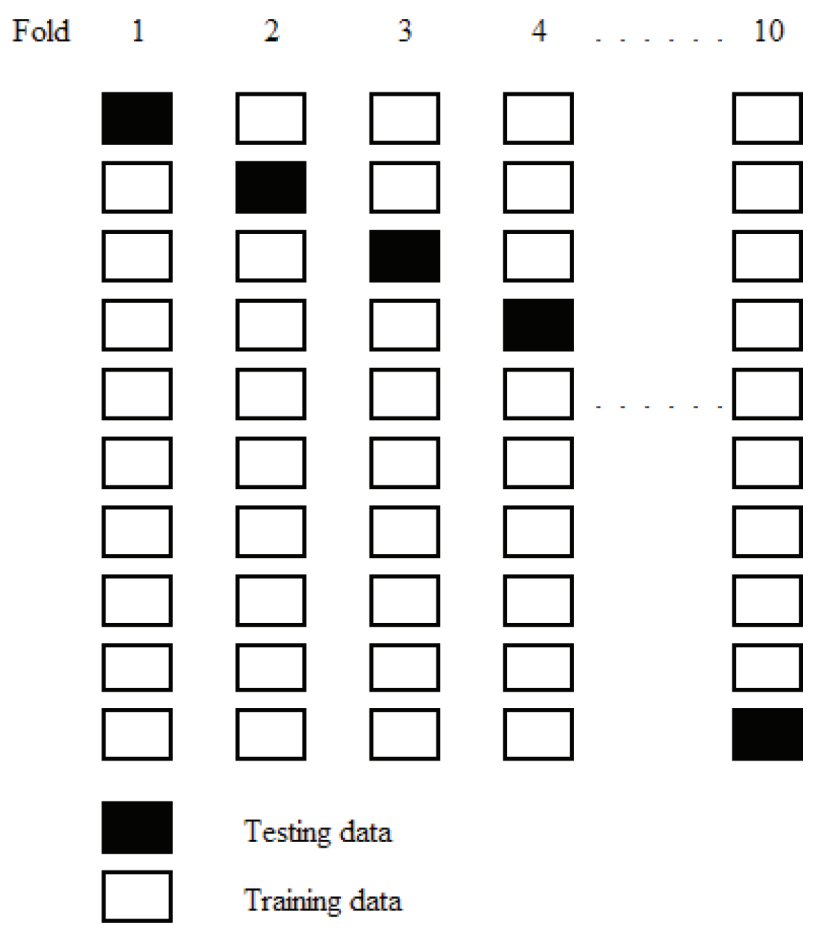

Fig. 7. 10-fold cross-validation

\section{Mean absolute percentage error}

Mean absolute percentage error (MAPE) is a measurement of prediction accuracy. It represents prediction percentage error. Small denominators can cause problems in MAPE value because small denominators generate large MAPE values that impact overall value. The MAPE is expressed using the following equation:

$$
M A P E=\frac{1}{n} \sum_{j=1}^{n}\left|\frac{y_{j}-\hat{y}_{j}}{y_{j}}\right| \times 100 \% .
$$

\section{Training time}

Training time represents time taken by the proposed model to train data and obtain the optimum prediction model.

To obtain an overall comparison, a normalized reference index (RI) (Chou et al. 2011) was created by combining the four performance measures (RMSE, MAE, and MAPE, and training time). The RI was obtained by calculating the average of each normalized performance measure. Performance measure values ranged from 1 (best) to 0 (worst). The equation of RI can be described as follows:

$$
R I=\frac{\sum_{i=1}^{n} \frac{x_{i}^{\max }-x_{i}}{x_{i}^{\max }-x_{i}^{\min }}}{n},
$$

where: $x_{i}$ is the measurement indicator (RMSE, MAPE, MAE, training time); $x_{i}^{\max }$ is the maximum value of the indicator among all prediction methods; $x_{i}^{\mathrm{min}}$ is the minimum value of the indicator among all prediction methods; $n$ is the number of measurement indicators.

\section{Results and discussion}

\subsection{Model performance}

A systematic methodology was previously established to calculate prediction performance. Database records contain several attributes related to productivity loss caused by change orders. Data preprocessing was done to improve data quality. In the data preprocessing stage, two kinds of analysis relate to attributes reduction methods. We performed Analyses 1 and 2 to compare the performance of each. Analysis 1 employed the CA method and Analysis 2 employed the PCA method, with each implementing training and testing processes in accordance with 10 -fold cross-validation.

In the testing process, each fold validates the performance of the proposed model. A comparison with other methods such as ESIM (Cheng, Wu 2009), ANN, and SVM was developed to show EFSIM as more accurate and reliable. Several performance measures (RMSE, MAE, MAPE, and training time) were employed to evaluate the proposed model.

Table 5 summarizes our comparison of Analyses 1 and 2 results. Optimal EFSIM parameter of Analysis 1 is $\mathrm{C}=31$ and $\gamma=0.574$, founded in fold 3. Meanwhile, $\mathrm{C}=31$ and $\gamma=0.566$ of fold 9 is regarded as the optimal EFSIM parameter in Analysis 2. In Analysis 1 earned better results in all EFSIM and ESIM performance measures except for MAPE. On the other hand, Analysis 2 obtained better results in SVM and ANN. Analysis 1 had a higher EFSIM training time than Analysis 2 because of its larger number of attributes.

However, the difference between the two analyses in terms of the MAPE performance measure was not significant. Table 5 shows EFSIM results found both Analysis 1 and Analysis 2 to be significantly more accurate than other AI techniques. Longer computation time is required for the EFSIM model due to the FL paradigm. The more attributes in a training process, the more training time is needed to obtain the prediction model.

Table 6 shows average Analysis 1 and 2 performance values. The best model with the smallest RMSE value is EFSIM (2.98\%). Moreover, Table 6 shows a

Table 5. Performance of predictive techniques for Analyses 1

\begin{tabular}{|c|c|c|c|c|c|}
\hline Analysis & $\begin{array}{l}\text { Predictive } \\
\text { technique }\end{array}$ & $\begin{array}{c}\text { RMSE } \\
(\%)\end{array}$ & $\begin{array}{c}\text { MAE } \\
(\%)\end{array}$ & $\begin{array}{c}\text { MAPE } \\
(\%)\end{array}$ & $\begin{array}{l}\text { Average } \\
\text { training } \\
\text { time (s) }\end{array}$ \\
\hline \multirow{4}{*}{$\begin{array}{l}\vec{n} \\
\frac{n}{\infty} \\
\frac{\pi}{3} \\
\frac{3}{4}\end{array}$} & EFSIM & 2.83 & 2.25 & 30.44 & 7065.73 \\
\hline & ESIM & 6.57 & 5.62 & 55.40 & 231.3 \\
\hline & ANN & 9.24 & 8.00 & 76.43 & 1 \\
\hline & SVM & 9.12 & 7.78 & 60.70 & 1 \\
\hline \multirow{4}{*}{ 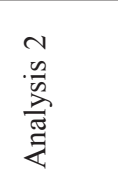 } & EFSIM & 3.13 & 2.51 & 23.85 & 5146.83 \\
\hline & ESIM & 7.12 & 6.07 & 48.12 & 138.2 \\
\hline & ANN & 8.79 & 7.55 & 72.82 & 1 \\
\hline & SVM & 8.61 & 7.23 & 65.16 & 1 \\
\hline
\end{tabular}
and 2 
direct relationship between RMSE and MAE. MAE values are always smaller than RMSE values. EFSIM obtains the smallest MAE value $(2.38 \%)$ of all models. MAPE values are inadequate in all models due to the series of small denominator values. MAPE values are consistent with other performance measures (RMSE and MAE). The best model is obtained by EFSIM (27.15\%).

Table 6. Average performance values of the predictive techniques

\begin{tabular}{lccccc}
\hline $\begin{array}{l}\text { Predictive } \\
\text { technique }\end{array}$ & $\begin{array}{c}\text { RMSE } \\
(\%)\end{array}$ & $\begin{array}{c}\text { MAE } \\
(\%)\end{array}$ & $\begin{array}{c}\text { MAPE } \\
(\%)\end{array}$ & $\begin{array}{c}\text { Average } \\
\text { training } \\
\text { time }(\mathrm{s})\end{array}$ & RI \\
\hline EFSIM & 2.98 & 2.38 & 27.15 & 6106.28 & 0.7500 \\
\hline ESIM & 6.84 & 5.84 & 51.76 & 184.75 & 0.5423 \\
\hline ANN & 9.01 & 7.77 & 74.62 & 1 & 0.2500 \\
\hline SVM & 8.86 & 7.70 & 62.93 & 1 & 0.3303 \\
\hline
\end{tabular}

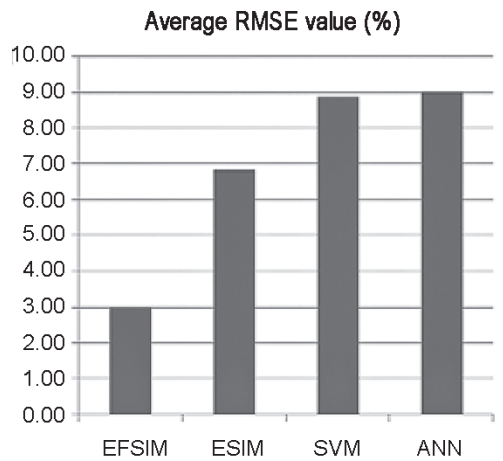

Average RMSE value
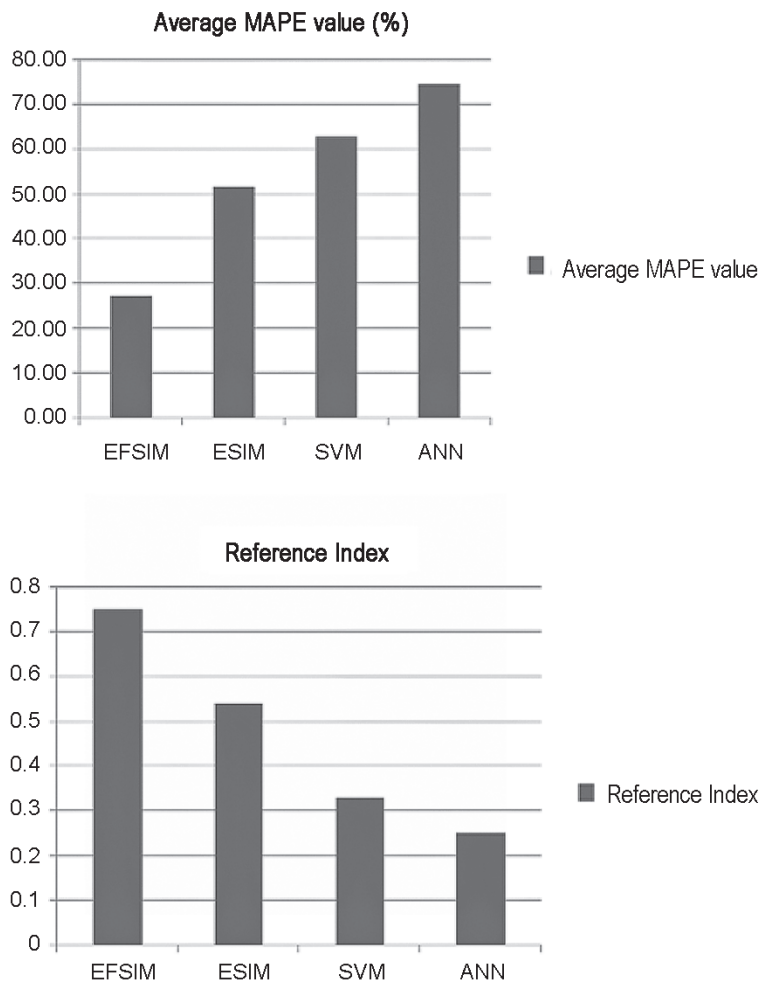

Reference Index

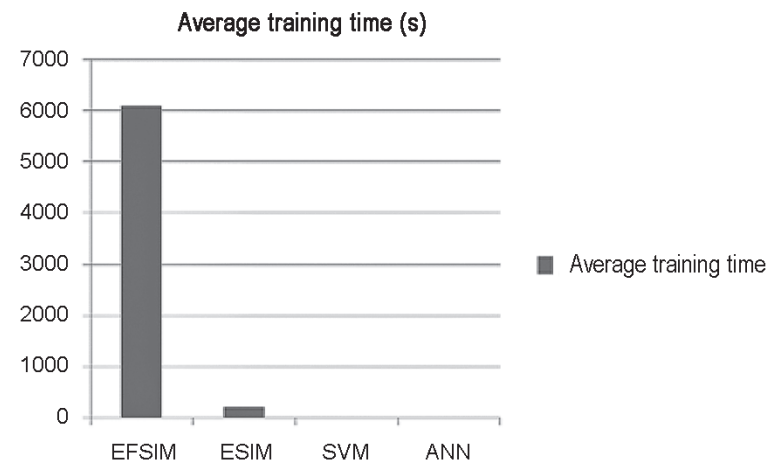

In terms of training time, both ANN and SVM train data relatively quickly, while ESIM requires more time and EFSIM requires the most time. This is due to the FL paradigm that requires more computational time during the training process and to the status of ESIM and EFSIM as hybrid AI techniques. Longer computational time is a trade off necessary to obtain greater accuracy. Figure 8 illustrates the performance described in Table 6 .

The normalized RI obtained a general measurement by combining all performance measures. Based on RI values, EFSIM is the best model, followed in order by ESIM, SVM, and ANN. Although EFSIM requires the longest training time, it consistently obtains the best results on most other performance measures. Thus, by fusing FL, SVM, and fmGA, EFSIM predicts changeorder-related productivity loss more accurately than the other models considered.

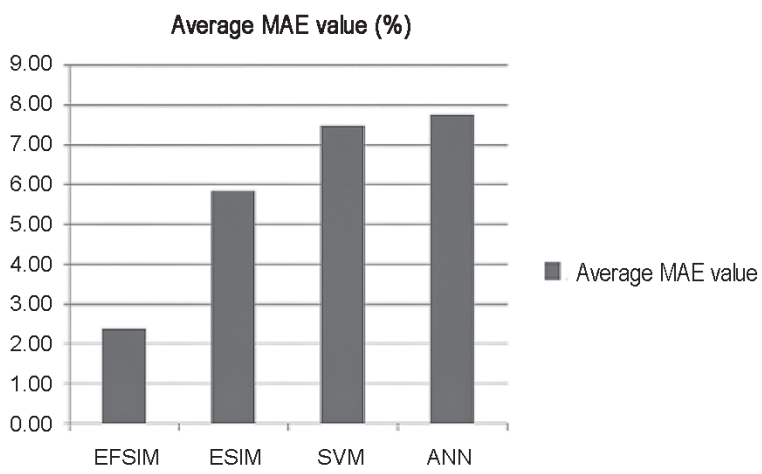

Fig. 8. Average result for performance measures with 10-fold cross-validation 


\subsection{Validation with previous studies}

We compared the performance of EFSIM against other methods such as the general regression model (Moselhi et al. 1991), electrical regression model (Hanna et al. 1999b), and neural network model (Moselhi et al. 2005). Our validation used a dataset of 33 records from Assem (2000) as training data and change-order data from the literature (Bruggink 1997) as testing data. Attributes used in this dataset were (1) timing impact of change orders $\left(\mathrm{TP}_{i}\right)$; (2) work type; and (3) type of impact (TI), which could be either change orders only or change orders plus 1 or 2 additional causes of productivity related impact. $\mathrm{TP}_{i}$ represents the ratio of actual change order hours to planned hours in each of the five construction periods ( $i=1$ to 5$)$ as shown in following equation:

$$
T P_{i}=\frac{H C O_{i}}{P H_{i}},
$$

where: $T P_{i}$ is the timing impact of change orders in period $i$; $\mathrm{HCO}_{i}$ is the actual change order hours during period $i ; \mathrm{PH}_{i}$ is the planned hours during period $i$; and $i$ is the period when change orders occur, where the value of $i$ can range from 1 to 5. This data set used NECA (1983) distribution for electrical work and the trapezoidal distribution of Bent and Thuman (1994) for other types of work to distribute the planned hours in each of the five construction periods.
The cases were analyzed using EFSIM and results were compared with previous studies found in Moselhi et al. (2005). Table 7 shows comparisons among all methods. Results demonstrate that the EFSIM model proposed in this study outperforms all other models in terms of estimating the impact of change orders on productivity. EFSIM obtained the smallest average error $(7.90 \%)$ and lowest average absolute error of any model. This shows that EFSIM improves prediction model accuracy and reliability.

\section{Conclusions}

This research proposes a hybrid AI technique, EFSIM, to predict productivity loss caused by change orders. The EFSIM, developed by fusing complementary AI techniques including FL, SVM, and fmGA, achieves prediction results superior to traditional techniques.

The developed model reduces the level of human intervention necessary to elicit MF shapes from questionnaire surveys and expert judgment; it also successfully identifies optimum penalty and kernel parameters. EFSIM is easy to apply and convenient for new users, and may be used by professionals without AI domain knowledge.

Test results show that EFSIM prediction performance is superior to other prediction methods such as ESIM, ANN, and SVM. Although EFSIM requires

Table 7. Actual versus estimated productivity loss (Moselhi et al. 2005)

\begin{tabular}{|c|c|c|c|c|c|c|c|c|c|}
\hline \multirow[b]{2}{*}{$\begin{array}{l}\text { Case No. } \\
\text { (j) }\end{array}$} & \multirow{2}{*}{$\begin{array}{c}\text { Actual } \\
\text { productivity } \\
\text { loss (h) }\end{array}$} & \multicolumn{2}{|c|}{ Moselhi et al. (1991) } & \multicolumn{2}{|c|}{ Hanna et al. (1999) } & \multicolumn{2}{|c|}{ Moselhi et al. (2005) } & \multicolumn{2}{|c|}{$\begin{array}{l}\text { Proposed model } \\
\text { (EFSIM) }\end{array}$} \\
\hline & & $\begin{array}{l}\text { Estimated } \\
\text { productivity } \\
\text { loss }(\mathrm{h})\end{array}$ & $\begin{array}{l}\text { Estimating } \\
\text { error }^{\mathrm{b}}(\%)\end{array}$ & $\begin{array}{c}\text { Estimated } \\
\text { productivity } \\
\text { loss (h) }\end{array}$ & $\begin{array}{l}\text { Estimating } \\
\text { error }^{\mathrm{b}}(\%)\end{array}$ & $\begin{array}{c}\text { Estimated } \\
\text { productivity } \\
\text { loss }(\mathrm{h})\end{array}$ & $\begin{array}{l}\text { Estimating } \\
\text { error }^{\mathrm{b}}(\%)\end{array}$ & $\begin{array}{l}\text { Estimated } \\
\text { productivity } \\
\text { loss }(\mathrm{h})\end{array}$ & $\begin{array}{l}\text { Estimating } \\
\text { error }^{\mathrm{b}}(\%)\end{array}$ \\
\hline 1 & 801 & 731 & 8.74 & 650 & 18.85 & 940 & -17.35 & 822 & -2.68 \\
\hline 2 & 1,073 & $1,050 \mathrm{a}$ & 2.14 & 713 & 33.55 & $1,245 \mathrm{a}$ & -16.03 & 1,113 & -3.75 \\
\hline 3 & 1,593 & 884 & 44.51 & 1,467 & 7.91 & 1,240 & 22.16 & 1,749 & -9.78 \\
\hline 4 & 1,741 & 2,070 & -18.90 & 2,041 & -17.23 & 2,674 & -53.59 & 1,799 & -3.32 \\
\hline 5 & 2,660 & 1,744 & 34.44 & 2,966 & -11.50 & 2,699 & -1.47 & 2,550 & 4.14 \\
\hline 6 & 5,139 & N/A & N/A & 2,485 & 51.64 & $5,078 \mathrm{a}$ & 1.19 & 4,912 & 4.42 \\
\hline 7 & 13,713 & 11,469 & 16.36 & 10,644 & 22.38 & 15,169 & -10.62 & 13,822 & -0.79 \\
\hline 8 & 10,575 & 5,073 & 52.03 & 2,438 & 76.95 & 7,077 & 33.08 & 8,350 & 21.04 \\
\hline \multicolumn{2}{|c|}{ Average error ${ }^{\mathrm{c}}(\%)$} & \multicolumn{2}{|c|}{30.45} & \multicolumn{2}{|c|}{40.50} & \multicolumn{2}{|c|}{17.83} & \multicolumn{2}{|c|}{7.90} \\
\hline \multicolumn{2}{|c|}{$\begin{array}{l}\text { Average absolute } \\
\text { error }^{\mathrm{d}}(\%)\end{array}$} & \multicolumn{2}{|c|}{25.30} & \multicolumn{2}{|c|}{30.00} & \multicolumn{2}{|c|}{19.44} & \multicolumn{2}{|c|}{6.24} \\
\hline
\end{tabular}

Notes: $\mathrm{N} / \mathrm{A}=$ not applicable (i.e. off model range);

${ }^{\text {a }}$ Two additional types of impact are considered in the estimate (i.e. TI $=3$ );

${ }^{\mathrm{b}}$ Estimating error $_{j}=\frac{\left(\text { Actual loss }_{j}-\text { Estimated loss }_{j}\right)}{\text { Actual loss }_{j}}$, where j represents case 1 to 8;

${ }^{c}$ Average error $=\frac{\sum_{j=1}^{8} \mid \text { Actual loss }_{j}-\text { Estimated loss }_{j} \mid}{\sum_{j=1}^{8} \text { Actual loss }_{j}} ;$

$\mathrm{d}$ Average absolute error $=\frac{\sum_{j=1}^{8} \mid \text { Estimated loss }_{j} \mid}{8}$. 
more computation time, EFSIM prediction results are significantly more accurate than competing methods. Validation results with previous studies in predicting the impact of change orders on productivity loss also indicate that EFSIM provides the smallest margin among competing methods. These results exhibit great potential for EFSIM as a tool to accurately predict change-order-related productivity loss. Moreover, the developed model manages to help the project manager to make an adjustment related to productivity loss caused by change order. Furthermore, this research paper succeed in demonstrating a hybrid Artificial Intelligence paradigm, FL-SVM-fmGA, for facilitating the decision making in the construction industry.

\section{References}

Assem, I. 2000. Estimating productivity losses due to change orders. Master's thesis. Concordia University, Montreal. 226 p.

Bent, J. A.; Thurman, A. 1994. Project management for engineering and construction. $2^{\text {nd }}$ ed. New Jersey: PrenticeHall Inc. 334 p.

Bojadziev, G.; Bojadziev, M. 2007. Fuzzy logic for business, finance, and management. $2^{\text {nd }} \mathrm{ed}$. Singapore: World Scientific. 232 p. http://dx.doi.org/10.1142/6451

Borra, S.; Di Ciaccio, A. 2010. Measuring the prediction error. A comparison of cross-validation, bootstrap and covariance penalty methods, Computational Statistics \&amp; Data Analysis 54(12): 2976-2989. http://dx.doi.org/10.1016/j.csda.2010.03.004

Bruggink, M. J. 1997. An investigation into the impacts of change orders on labor efficiency in the electrical construction industry. Master's thesis. University of Wisconsin-Madison, Madison. 318 p.

Burges, C. J. C. 1998. A tutorial on support vector machines for pattern recognition, Data Mining and Knowledge Discovery 2(2): 121-167.

http://dx.doi.org/10.1023/A:1009715923555

Cheng, M.-Y.; Roy, A. F. V. 2010. Evolutionary fuzzy decision model for construction management using support vector machine, Expert Systems with Applications 37(8): 6061-6069. http://dx.doi.org/10.1016/j.eswa.2010.02.120

Cheng, M.-Y.; Wu, Y.-W. 2009. Evolutionary support vector machine inference system for construction management, Automation in Construction 18(5): 597-604. http://dx.doi.org/10.1016/j.autcon.2008.12.002

Chou, J.-S.; Chiu, C.-K.; Farfoura, M.; Al-Taharwa, I. 2011. Optimizing the prediction accuracy of concrete compressive strength based on a comparison of data-mining techniques, Journal of Computing in Civil Engineering 25(3): 242-253. http://dx.doi.org/10.1061/(ASCE)CP.1943-5487.0000088

Deb, K.; Goldberg, D. E. 1991. $m G A$ in $C$ : a messy genetic algorithm. C. IllGAL technical report 91008. Urbana, Illinois, University of Illinois at Urbana-Champaign. 118 p.

Feng, C.-W.; Wu, H.-T. 2006. Integrating fmGA and CYCLONE to optimize the schedule of dispatching RMC trucks, Automation in Construction 15(2): 186-199. http://dx.doi.org/10.1016/j.autcon.2005.04.001

Goldberg, D.; Deb, K.; Kargupta, H.; Harik, G. 1993. Rapid accurate optimization of difficult problems using fast messy genetic algorithms, in Proceedings of the $5^{\text {th }}$ International Conference on Genetic Algorithms, 17-21 July 1993, University of Illinois, Morgan Kaufmann Publishers Inc, 56-64.

Han, J.; Kamber, M. 2007. Data mining: concept and techniques. $2^{\text {nd }}$ ed. San Fransisco: Morgan Kaufmann Publisher. $800 \mathrm{p}$.
Hanna, A. S.; Lotfallah, W. B.; Lee, M.-J. 2002. Statisticalfuzzy approach to quantify cumulative impact of change orders, Journal of Computing in Civil Engineering 16(4): 252-258.

http://dx.doi.org/10.1061/(ASCE)0887-3801(2002)16:4(252)

Hanna, A. S.; Russell, J. S.; Gotzion, T. W.; Nordheim, E. V. 1999a. Impact of change orders on labor efficiency for mechanical construction, Journal of Construction Engineering and Management 125(3): 176-184.

http://dx.doi.org/10.1061/(ASCE)0733-9364(1999)125:3(176)

Hanna, A. S.; Russell, J. S.; Nordheim, E. V.; Bruggink, M. J. 1999b. Impact of change orders on labor efficiency for electrical construction, Journal of Construction Engineering and Management 125(4): 224-232.

http://dx.doi.org/10.1061/(ASCE)0733-9364(1999)125:4(224)

Hester, W. T.; Kuprenas, J. A.; Chang, T. C. 1991. Construction changes and change orders: their magnitude and impact. Austin: Construction Industry Institute. $125 \mathrm{p}$.

Hsu, C.-W.; Chang, C.-C.; Lin, C.-J. 2003. A practical guide to support vector classification. Taipei, Taiwan, National Taiwan University. $16 \mathrm{p}$.

Ibbs, W. 2005. Impact of change's timing on labor productivity, Journal of Construction Engineering and Management 131(11): 1219-1223.

http://dx.doi.org/10.1061/(ASCE)0733-9364(2005)131:11(1219)

Ishigami, H.; Fukuda, T.; Shibata, T.; Arai, F. 1995. Structure optimization of fuzzy neural network by genetic algorithm, Fuzzy Sets and Systems 71(3): 257-264.

http://dx.doi.org/10.1016/0165-0114(94)00283-D

Keane, P.; Sertyesilisik, B.; Ross, A. D. 2010. Variations and change orders on construction projects, Journal of Legal Affairs and Dispute Resolution in Engineering and Construction 2(2): 89-96.

http://dx.doi.org/10.1061/(ASCE)LA.1943-4170.0000016

Ko, C. H. 2002. Evolutionary fuzzy neural inference model (EFNIM) for decision-making in construction management. PhD dissertation. National Taiwan University of Science and Technology, Taipei, Taiwan. 205 p.

Leonard, C. A. 1988. The effects of change orders on productivity. MS thesis. Concordia University, Montreal, Quebec. $156 \mathrm{p}$.

Martinez, C.; Castillo, O.; Montiel, O. 2008. Comparison between ant colony and genetic algorithms for fuzzy system optimization, Soft Computing for Hybrid Intelligent Systems 154: 71-86. http://dx.doi.org/10.1007/978-3-540-70812-4 5

Moselhi, O.; Assem, I.; El-Rayes, K. 2005. Change orders impact on labor productivity, Journal of Construction Engineering and Management 131(3): 354-359.

http://dx.doi.org/10.1061/(ASCE)0733-9364(2005)131:3(354)

Moselhi, O.; Leonard, C.; Fazio, P. 1991. Impact of change orders on construction productivity, Canadian Journal of Civil Engineering 18(3): 484-492. http://dx.doi.org/10.1139/191-059

National Electrical Contractor's Association (NECA). 1983. Rate of manpower consumption in electrical construction. Electrical Construction Peak Workforce Report: 5. Washington DC.

Shahi, A.; Atan, R. B.; Sulaiman, M. N. 2009. An effective fuzzy C-mean and type-2 fuzzy logic for weather forecasting, Journal of Theoritical and Applied Information Technology 5(5): 556-567.

Vapnik, V. N. 1995. The nature of statistical learning theory. New York: Springer-Verlag. 188 p. http://dx.doi.org/10.1007/978-1-4757-2440-0

Yongqiao, W.; Shouyang, W.; Lai, K. K. 2005. A new fuzzy support vector machine to evaluate credit risk, IEEE Transactions on Fuzzy Systems 13(6): 820-831. http://dx.doi.org/10.1109/TFUZZ.2005.859320 
Zhang, S.; Zhang, C.; Yang, Q. 2003. Data preparation for data mining, Applied Artificial Intelligence 17(5-6): 375-381. http://dx.doi.org/10.1080/713827180

Min-Yuan CHENG. He is Distinguished Professor in the Department of Civil and Construction Engineering at the National Taiwan University of Science and Technology, Taipei, Taiwan. He received his PhD degree from University of Texas at Austin, USA. He has about 20 years of teaching and research experience. He has authored about over 100 research papers published in various reputed international journal and conference proceedings. His research interests include: construction business process re-engineering, geographic information system, construction automation \& e-business for construction management, project management information system, and artificial intelligence applications in construction management.

Dedy Kurniawan WIBOWO. He joined Dual Degree Master Program between Sepuluh Nopember Institute of Technology, Indonesia and National Taiwan University of Science and Technology, Taiwan in 2010. He received his dual MT and MSc degree in 2012. His area of expertise is construction management.

Doddy PRAYOGO. He is a PhD student in the Department of Civil and Construction Engineering at the National Taiwan University of Science and Technology, Taipei, Taiwan. He received his ST degree in Petra Christian University, Indonesia, his dual MT and MSc degree in Sepuluh Nopember Institute of Technology, Indonesia and National Taiwan University of Science and Technology, Taiwan. He has authored several research papers published in various international journals and conference proceedings. His PhD research is focused on the artificial intelligence applications in construction management.

Andreas F. V. ROY. He is a Lecturer in the Department of Civil Engineering, Faculty of Engineering, Parahyangan Catholic University. He is a member of ASCE 481587 . His research interests include: infrastructure maintenance management systems, decision \& risk analysis, management information system and applications of artificial intelligence in construction engineering and management. 